

\title{
Dispositif de mesure des températures au four solaire par pyrométrie dans l'ultra-violet
}

Marc Foex, Jean-Pierre Coutures

\section{To cite this version:}

Marc Foex, Jean-Pierre Coutures. Dispositif de mesure des températures au four solaire par pyrométrie dans l'ultra-violet. Revue de Physique Appliquée, 1969, 4 (3), pp.381-382. 10.1051/rphysap:0196900403038100 . jpa-00243299

\section{HAL Id: jpa-00243299 https://hal.science/jpa-00243299}

Submitted on 1 Jan 1969

HAL is a multi-disciplinary open access archive for the deposit and dissemination of scientific research documents, whether they are published or not. The documents may come from teaching and research institutions in France or abroad, or from public or private research centers.
L'archive ouverte pluridisciplinaire HAL, est destinée au dépôt et à la diffusion de documents scientifiques de niveau recherche, publiés ou non, émanant des établissements d'enseignement et de recherche français ou étrangers, des laboratoires publics ou privés. 


\title{
DISPOSITIF DE MESURE DES TEMPÉRATURES AU FOUR SOLAIRE PAR PYROMÉTRIE DANS L'ULTRA-VIOLET
}

\author{
Par Marc FOEX et Jean-Pierre COUTURES, \\ Laboratoire des Ultra-Réfractaires, C.N.R.S., B.P. $\mathrm{n}^{0}{ }_{5}$, 66-Odeillo. \\ (Reçu le 14 mars 1969.)
}

\begin{abstract}
Résumé. - Le présent article décrit le principe de mesure des températures et des facteurs d'émission monochromatique apparents, de produits traités au moyen de fours solaires ou de fours à image par l'utilisation de pyromètres munis de filtres interférentiels opérant dans l'ultra-violet, et son application à l'analyse thermique.
\end{abstract}

\begin{abstract}
This paper describes the principle of the temperature and apparent monochromatic emissive power measurements of products treated with solar or imaging furnaces by the utilisation of electronic pyrometer providing interferences filters to pass radiation in the near ultra-violet region. A typical application on thermal analysis is described.
\end{abstract}

La mesure des températures des produits directement traités au foyer des fours solaires présente quelques difficultés par suite de la réflexion d'une partie du rayonnement du soleil par ces produits, ainsi que par la méconnaissance de leur facteur d'émission dans les conditions de l'expérience [1 à 3$]$.

Afin de pallier le premier de ces inconvénients, on utilise un pyromètre fonctionnant dans l'ultra-violet (filtre interférentiel), cependant que le rayonnement solaire incident est, avant son arrivée sur le miroir parabolique, soumis à l'action d'un filtre arrêtant l'ultra-violet et laissant passer le rayonnement de plus grande longueur d'onde. Ce filtre doit couvrir entièrement le miroir parabolique. Les pyromètres possédant un photomultiplicateur comme détecteur de rayonnement conviennent bien à cet usage. Les filtres sont en matière plastique, comportant des charges anti-ultraviolettes incolores ou légèrement colorantes (jaunes); ils peuvent être aussi revêtus de laques spéciales.

D'autre part, c'est dans le domaine de l'ultra-violet que le rayonnement solaire se différencie le plus du rayonnement émis par les produits traités au foyer des fours solaires ; ces derniers, en général portés à des températures comprises entre $2000{ }^{\circ} \mathrm{C}$ et $3000^{\circ} \mathrm{C}$, ne dépassent que très rarement $3500^{\circ} \mathrm{C}$. Ainsi vers 0,36-0,37 micron, le rayonnement émis par le produit ne dépasse pas $0,2 \mathrm{~W} / \mathrm{cm}^{2}$ par micron à $2000^{\circ} \mathrm{C}$ et 30 à $40 \mathrm{~W} / \mathrm{cm}^{2}$ par micron à $3000^{\circ} \mathrm{C}$, alors que l'éclairement réalisé par le soleil au foyer est de l'ordre de $500 \mathrm{~W} / \mathrm{cm}^{2}$ par micron.

Il est facile de tirer parti de cette différence pour mesurer le facteur d'émission en déterminant la fraction de rayonnement solaire réfléchie par le produit, et cela pour la même longueur d'onde que celle utilisée pour la mesure de température.
Il est très important, afin que la température du produit n'évolue pas ou évolue aussi faiblement que possible, que l'énergie globale admise au niveau du produit ne soit pas changée sensiblement lors de l'admission du rayonnement riche en ultra-violet. Dans le même esprit, la répartition énergétique du rayonnement parvenant au produit devra être aussi peu modifiée que possible à ce stade de la mesure. On devra donc ne laisser passer en dehors des filtres couvrant le miroir parabolique qu'un flux de lumière solaire assez réduit, de façon à ce que les rayonnements émis et reçus dans l'ultra-violet soient par exemple d'un ordre de grandeur voisin. Dans le cas de mesures effectuées dans l'ultra-violet, ce flux sera très réduit lors des mesures réalisées en dessous de $3000^{\circ} \mathrm{C}$. Des petits filtres laissant ou non passer l'ultra-violet seront disposés sur l'ouverture réalisée dans les écrans.

Avec le même pyromètre muni de son filtre interférentiel (ultra-violet), on procède successivement aux trois mesures suivantes :

1) Température apparente du produit en présence du rayonnement filtré et en l'absence du rayonnement ultra-violet ;

2) Température apparente du produit en présence du rayonnement filtré et d'une fraction déterminée du rayonnement ultra-violet du soleil;

3) Température apparente d'un corps étalon, par exemple magnésie blanche, présentant un facteur pratique d'émission bien connu soumis à l'action de la même fraction déterminée du rayonnement ultraviolet du soleil. De l'ensemble de ces mesures il est facile de déduire le facteur d'émission apparent de la substance traitée ainsi que sa température vraie. 
Les miroirs en verres argentés absorbent une partie notable de l'ultra-violet; le maximum d'absorption de l'argent métallique se situant à 0,316 micron, il peut

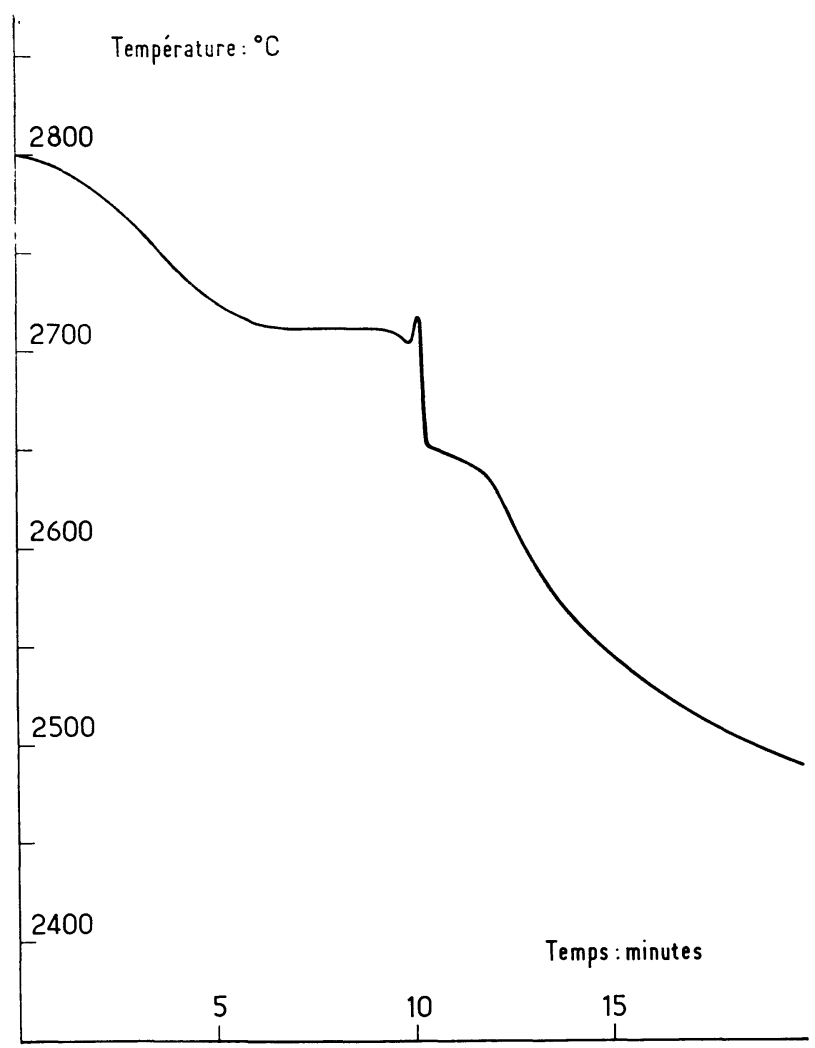

FIG. 1. - Courbe température-temps obtenue après fusion au four solaire de la zircone et occultation progressive $\mathrm{du}$ rayonnement incident. donc être intéressant d'utiliser un filtre utilisant cette longueur d'onde. Cependant, il est difficile dans ce cas de procéder aux corrections de facteur d'émission par la méthode précédente. On devra donc préférer utiliser des longueurs d'ondes plus proches du visible ou même situées dans le visible très court. L'emploi du pyromètre fonctionnant dans le vert est même possible, mais dans ce cas on devra utiliser des filtres absorbant une grande partie du rayonnement solaire, ce qui limite beaucoup les performances, en température, de ces appareils. Les miroirs d'aluminium ou aluminisés ne présentent pas les inconvénients précédents.

Enfin, il convient de noter que le dispositif employé ici est applicable à la plupart des fours à image, tout au moins dans son principe. En effet, beaucoup de ces derniers appareils sont beaucoup plus condensés que les fours solaires, et les filtres sont de ce fait soumis à des éclairements énergétiques beaucoup plus importants.

A titre d'exemple et après correction du facteur d'émission apparent (0,366 micron), on a donné ( fig. 1) la courbe de refroidissement d'une masse de zircone d'une vingtaine de grammes fondue avec un four solaire de $2 \mathrm{~kW}$. Le produit placé dans un four tournant est centrifugé de façon à créer une cavité $(35 \mathrm{~mm}$ de profondeur, $10 \mathrm{~mm}$ de diamètre) dont l'ouverture est placée au foyer et dont le fond présente une température homogène. La correction de « facteur d'émission apparent » est ainsi beaucoup plus réduite (elle correspond à quelques degrés au niveau de la fusion) que lorsqu'on examine un produit dont la surface est directement placée au foyer. Afin de procéder au refroidissement, on obture progressivement le rayonnement solaire au moyen d'un rideau. On note un palier très net correspondant à la solidification de la zircone, la valeur observée $\left(2710^{\circ} \mathrm{C}\right)$ est en bon accord avec les données de la littérature.

\section{BIBLIOGRAPHIE}

[1] FOEX (M.), Colloque sur l'Énergie Solaire, Montlouis, 1958 ; édition du C.N.R.S., p. 255, Paris, 1961.

[2] FoEx (M.), Revue Internationale des Hautes Températures et des Réfractaires, 1966, 3, 326 .

[3] FoEx (M.), Colloque sur la Magnétohydrodynamique, Varsovie, 1968. 